\title{
Aleitamento materno e o papel do enfermeiro
}

\author{
Breastfeeding and the Role of the Nurses \\ Lactancia materna y el papel de la enfermera
}

Recebido: 01/06/2021 | Revisado: 07/06/2021 | Aceito: 09/06/2021 | Publicado: 23/06/2021

\author{
Cinthya Posley Aguiar de Oliveira \\ ORCID: https://orcid.org/0000-0001-8795-4365 \\ Faculdade Independente do Nordeste, Brasil \\ E-mail: Cinthya.posley@gmail.com \\ Julia Souza Santos Nunes \\ ORCID: https://orcid.org/0000-0001-6180-5791 \\ Faculdade Independente do Nordeste, Brasil \\ E-mail: juliassenf@tahoo.com.br
}

\begin{abstract}
Resumo
O aleitamento materno é essencial para a nutrição, desenvolvimento e crescimento saudável da criança e para a mãe também existem grandes benefícios, este ato deve ser algo prazeroso, durante a amamentação forma-se um grande elo entre mãe e filho, mas para que esse elo se forme é necessário que a mãe se sinta segura e confortável, e que a criança sugue o leite adequadamente uma pega errada pode provocar um grande desconforto para a mãe causando assim um trauma na hora da amamentação o que ocasionar um desmame precoce, e para que isso não aconteça o enfermeiro tem um papel importante, na orientação e auxílio tanto no pré-natal e logo após o nascimento da criança. O estudo tem como objetivo analisar a atuação do(a) enfermeiro(a) na promoção, incentivo e apoio às mães no aleitamento materno. Trata-se de uma pesquisa de revisão integrativa que foi realizada por uma revisão bibliográfica baseada em obras secundárias que abordam a temática, publicadas no período de 2015 a 2020. Os resultados encontrados ressaltam a importância do enfermeiro frente ao cuidado, orientação e auxílio às gestantes e puérpera no aleitamento materno. Esta revisão nos mostra o quanto a presença do enfermeiro é essencial, que seu conhecimento teórico, seu olhar humano e capacitação como profissional se tornam imprescindíveis para estas mães e seus bebês.
\end{abstract}

Palavras-chave: Aleitamento materno; Alimentação infantil; Papel do enfermeiro.

\begin{abstract}
Mother breast-feeding is essential for the child's nutrition, development and healthy growth, and for the mother there are also great benefits, this act should be something pleasurable, during mother breast-feeding a great connection between mother and child is formed, but for that connection to be formed it is necessary for the mother to feel safe and comfortable, and for the child to suck the milk properly, the wrong handle can cause great discomfort for the mother, thus causing trauma at the time of mother breast-feeding, which can cause early weaning, so to prevent this to happen the nurse has an important performance in the guidance and assistance both in prenatal care and immediate post-birth. The study aims to analyze the performance of nurses in promoting, encouraging and supporting mothers in breastfeeding. This is an integrative review research that will be carried out by a literature review based on secondary works that address the theme, published in the period from 2015 to 2020. The results found underscore the importance of the nurse concerning the care, guidance and assistance to pregnant women and puerperal women in breastfeeding, this review shows us how essential the presence of nurses is, that their technical and scientific knowledge his human look and training as a professional become essential for these mothers and their babies.
\end{abstract}

Keywords: Mother breast-feeding; Infant feeding; Nurse performance.

\section{Resumen}

La lactancia materna es fundamental para la nutrición, desarrollo y crecimiento sano del niño, pero también hay grandes beneficios para la madre, como el de crear una importante conexión con su bebé. Para que se forme ese vínculo, es necesario que la lactancia sea de forma correcta. Primero que todo la madre se tiene que sentir segura y cómoda para que el niño succione la leche correctamente, el mal manejo puede ocasionar un gran malestar a ella, provocando así traumatismos, lo que puede provocar un destete precoz. Para evitar que esto suceda la enfermera tiene un importante desempeño en la orientación y asistencia tanto en el cuidado prenatal como en el posparto inmediato. El estudio tiene como objetivo analizar el desempeño de las enfermeras en la promoción, incentivo y apoyo a las madres en la lactancia materna. Se trata de una investigación de revisión integradora que se llevará a cabo mediante una revisión de la literatura a partir de trabajos secundarios que abordan la temática, publicados en el período de 2015 a 2020. Los resultados encontrados subrayan la importancia del enfermero en la atención, orientación y asistencia a mujeres embarazadas y puérperas en la lactancia. Esta revisión nos muestra lo esencial e importante para las madres y sus bebés la presencia de enfermeras, ya que aportan con conocimientos técnicos, científicos, su mirada humana y su formación profesional.

Palabras clave: Lactancia materna; Alimentación infantil; Importancia de la enfermera. 


\section{Introdução}

O aleitamento materno é a melhor e mais eficaz estratégia natural de vínculo, afeto, proteção e nutrição para a criança trazendo assim inúmeras vantagens para esta e sua mãe. Para o recém-nascido as vantagens vão desde a proteção respiratória e para a mãe uma possível proteção contra o câncer de mama e ovário. Proporciona o ganho adequado de peso e ele é isento de contaminação promovendo assim proteção imunológica para a criança, devido leite materno possui substâncias que beneficiam o sistema imunológico da criança, como anticorpos, fatores imunes, enzimas e células brancas do sangue e por ser repleto de nutrientes que são capazes de suprir qualquer necessidade alimentar da criança até os seis meses de vida, após essa idade deve ser feito um complemento alimentar com alimentos adequados a que atenderão as necessidades nutricionais neste período, prevenindo assim a morbimortalidade infantil. Além disso, a amamentação estimula o vínculo afetivo entre mãe e filho (Costa, Silva, Machado, Soares, Brezolin \& Silva, 2019).

A Organização Mundial de Saúde (OMS, 2012) recomenda a prática do aleitamento materno até os dois anos ou mais, devendo ser exclusiva nos primeiros seis meses de vida. A inserção de práticas e iniciativas dos órgãos governamentais e não governamentais à assistência da amamentação é um dos fatores importantes na promoção à saúde materno-infantil, no entanto, algumas mães encontram dificuldades para essa assistência especializada. A assistência ao aleitamento consistiu-se no ensino de técnicas simplificadas de manejo do aleitamento.

Crianças que foram alimentadas com leite materno desenvolvem anticorpos que fazem com que a ida ao médico, hospitalização e uso de medicamentos sejam diminuídas, tendo em vista que crianças que amamentaram com leite materno adoecem menos, isso também diminui o absenteísmo dos pais ao trabalho (Brasil, 2015)

O papel do enfermeiro e da equipe multidisciplinar é essencial para o auxílio e orientação dessas mães, não basta apenas informar e educar e sim orientar de maneira correta o manejo e pega do peito para proporcionar à criança uma alimentação adequada e para a mãe um momento prazeroso e com eficácia. O processo de amamentação embora de aparente simplicidade requer um complexo conjunto de condições interacionais no contexto social da mulher e seu filho (Alves, Carvalho, Lopez, Silva, Teixeira, 2018).

O intuito desta revisão de literatura é relatar a importância da amamentação para a saúde, desenvolvimento e crescimento saudável da criança, para a mãe uma sensação prazerosa e agradável e quanto ao enfermeiro, este tem papel primordial neste momento, orientar, auxiliar e ajudar a mãe nesse novo momento de sua vida. O presente estudo tem como objetivo analisar a atuação do (a) enfermeiro (a) na promoção, incentivo e apoio às mães no aleitamento materno. Tendo em vista a necessidade de uma orientação de um profissional qualificado e que este é um dos maiores contatos entre mãe e filho.

\section{Metodologia}

Trata-se de uma pesquisa de revisão integrativa que será realizada por uma revisão bibliográfica baseada em obras secundárias que abordam a temática, publicadas no período de 2015 a 2020. Segundo Souza (2010), podemos usar o método de revisão integrativa para aplicarmos os resultados dos estudos e polos em prática. (Souza, Silva \& Carvalho, 2010).

Para a formulação da revisão integrativa apresentam-se as seis fases do processo de elaboração: identificação do tema e elaboração da questão de pesquisa, amostragem ou pesquisa da literatura, colheita de dados, análise crítica dos estudos incluídos, interpretação e discussão dos resultados e apresentação da revisão/síntese de conhecimento e conclusões (Souza, Silva \& Carvalho 2010).

Segundo Ercole (2014), denomina-se integrativa pelo fato das informações obtidas poderem ser usadas de diversas maneiras para que seu resultado seja melhor aproveitado. (Ercole, Melo \& Alcoforado, 2014). Para o levantamento dos artigos na literatura foi realizada uma busca nas seguintes bases de dados: Literatura Latino-Americana e do Caribe em Ciências da Saúde (LILACS), Revista da Enfermagem, Portal Regional da Biblioteca Virtual de Saúde (BVS) e Scientific Eletronic 
Library Online (Scielo), Com os seguintes descritores: Aleitamento materno, Cuidado de Enfermagem, Enfermeiros quais fazem parte dos Descritores em Ciências da Saúde (DeCS).

\section{Resultados}

No processo de seleção dos artigos para estudo foram encontrados 35 artigos e utilizados somente 7, foram excluídos 28 artigos dos quais não se adequaram à temática proposta.

Os artigos selecionados foram organizados em um quadro, seguindo a distribuição: ano de publicação, autores, título, objetivos e principais resultados, conforme segue abaixo:

Quadro 1 - Distribuição dos artigos científicos segundo ano de publicação, autores, título, objetivos e principais resultados.

\begin{tabular}{|c|c|c|c|c|}
\hline ANO & UTORES & TÍTULOS & OBJETIVOS & PRINCIPAIS RESULTADOS \\
\hline 2019 & $\begin{array}{l}\text { Costa, Felipe dos Santos; Silva, } \\
\text { Jorge Luiz Lima da; Machado, } \\
\text { Emanoele Amaral; Soares, } \\
\text { Lunna Machado; Brezolin, } \\
\text { Cristian Antônio; Silva, João } \\
\text { Victor Lima. }\end{array}$ & $\begin{array}{l}\text { Promoção do } \\
\text { aleitamento } \\
\text { materno no } \\
\text { contexto da } \\
\text { estratégia de saúde } \\
\text { da família }\end{array}$ & $\begin{array}{l}\text { Descrever a promoção } \\
\text { da saúde para } \\
\text { o aleitamento } \\
\text { materno e refletir sobre } \\
\text { sua importância no } \\
\text { espaço da Estratégia de } \\
\text { Saúde da Família, onde } \\
\text { os contatos com a } \\
\text { gestante são } \\
\text { oportunizados. }\end{array}$ & $\begin{array}{l}\text { Informar as futuras mães a importância da } \\
\text { amamentação educar e sensibilizar a respeito das } \\
\text { práticas de amamentação. } \\
\text { Ações educativas e assistenciais em patologias } \\
\text { mamárias que levam ao desmame precoce. } \\
\text { Planejamento e redirecionamento das políticas } \\
\text { setoriais na área de saúde da mulher e da criança, } \\
\text { volta. } \\
\text { Ações comunitárias de promoção à saúde acerca da } \\
\text { amamentação e seus aspectos. }\end{array}$ \\
\hline 2019 & $\begin{array}{c}\text { Lima, Camila Mesquita } \\
\text { de; Sousa, Leilane Barbosa } \\
\text { de; Costa, Edmara } \\
\text { Chaves; Santos, Marks } \\
\text { Passos; Cavalcanti, Marianna } \\
\text { Carvalho e Souza Leão; Maciel, } \\
\text { Nathanael de Souza. }\end{array}$ & $\begin{array}{l}\text { Auto eficácia na } \\
\text { amamentação } \\
\text { exclusia: } \\
\text { avaliação dos } \\
\text { domínios técnica e } \\
\text { pensamentos } \\
\text { intrapessoais em } \\
\text { puérperas. }\end{array}$ & $\begin{array}{l}\text { Avaliar } \\
\text { a autoeficácia de } \\
\text { puérperas } \\
\text { na amamentação } \\
\text { exclusiva. }\end{array}$ & $\begin{array}{l}\text { Observou a eficácia das orientações e informações } \\
\text { ofertadas para as puérperas em relação à } \\
\text { amamentação exclusiva. } \\
\text { O enfermeiro tem um papel relevante pois ele é o } \\
\text { profissional que mais se relaciona e estreita laços com } \\
\text { a nutrise desta forma suas orientações e intervenção } \\
\text { torna-se fundamental para o acolhimento desta mães. }\end{array}$ \\
\hline 2019 & $\begin{array}{l}\text { Souza, Rosangela de Mattos } \\
\text { Pereira de; Alves, Valdecyr } \\
\text { Herdy; Rodrigues, Diego } \\
\text { Pereira; Branco, Maria Bertilla } \\
\text { Lutterbach Riker; Oliveira, } \\
\text { Fernanda Lopes de; Santos, } \\
\text { Márcia Vieira dos. }\end{array}$ & $\begin{array}{l}\text { O conhecimento do } \\
\text { enfermeiro acerca } \\
\text { do manejo clínico } \\
\text { da amamentação: } \\
\text { saberes e práticas. }\end{array}$ & $\begin{array}{c}\text { Analisar } \\
\text { o conhecimento } \\
\text { dos enfermeiros na } \\
\text { realização } \\
\text { das estratégias para o } \\
\text { manejo clínico } \\
\text { da amamentação. }\end{array}$ & $\begin{array}{l}\text { Reconhecimento da capacitação do enfermeiro ao } \\
\text { orientar as puérperas sobre a importância do } \\
\text { aleitamento. } \\
\text { A capacitação do enfermeiro é fundamental para o } \\
\text { desenvolvimento de métodos que orientem e auxiliam } \\
\text { as mães durante a amamentação é crucial para um } \\
\text { bom manejo clínico. }\end{array}$ \\
\hline 2018 & $\begin{array}{l}\text { Costa, Evelyn Farias Gomes } \\
\quad \text { da; Alves, Valdecyr } \\
\text { Herdy; Souza, Rosangela de } \\
\text { Mattos Pereira de; Rodrigues, } \\
\text { Diego Pereira; Santos, Márcia } \\
\text { Vieira dos; Oliveira, Fernanda } \\
\quad \text { Lopes de }\end{array}$ & $\begin{array}{l}\text { Atuação do } \\
\text { enfermeiro no } \\
\text { manejo clínico da } \\
\text { amamentação: } \\
\text { estratégias para o } \\
\text { aleitamento } \\
\text { materno }\end{array}$ & $\begin{array}{l}\text { Compreender } \\
\text { as estratégias de orienta } \\
\text { ção realizada } \\
\text { pelos enfermeiros dura } \\
\text { nte o processo do } \\
\text { manejo clínico } \\
\text { da amamentação. }\end{array}$ & $\begin{array}{l}\text { O apoio no manejo clínico do aleitamento materno na } \\
\text { perspectiva do cuidar; o apoio técnico-prático do } \\
\text { manejo clínico da amamentação; e o manejo clínico } \\
\text { da amamentação a } \\
\text { da orientação dos enfermeiros. }\end{array}$ \\
\hline 2018 & $\begin{array}{l}\text { Alves, Tássia Regine de } \\
\text { Morais; Carvalho, Jovanka } \\
\text { Bittencourt Leite de; Lopes, } \\
\text { Thais Rosental Gabriel; Silva, } \\
\text { Glauber Weder dos } \\
\text { Santos; Teixeira, Gracimary } \\
\text { Alves }\end{array}$ & $\begin{array}{l}\text { Contribuições de } \\
\text { enfermeiros na } \\
\text { promoção do } \\
\text { aleitamento } \\
\text { materno exclusivo }\end{array}$ & $\begin{array}{c}\text { Identificar as } \\
\text { contribuições } \\
\text { de enfermeiros na } \\
\text { promoção } \\
\text { do aleitamento } \\
\text { materno exclusivo }\end{array}$ & $\begin{array}{l}\text { O papel relevante do enfermeiro frente ao processo de } \\
\text { amamentação exclusiva visando a promoção de } \\
\text { atividades de educação em saúde durante o ciclo } \\
\text { gravídico puerperal. } \\
\text { Educação através da assistência no pré-natal, } \\
\text { fortalecimento do vínculo através de visitas } \\
\text { domiciliares, apoio e orientação para que a mãe } \\
\text { planeje sua autonomia e desenvolva sua confiança na } \\
\text { hora da amamentação. }\end{array}$ \\
\hline 2020 & $\begin{array}{c}\text { Silva, Luana Santiago da; Leal, } \\
\text { Natália Pessoa da } \\
\text { Rocha; Pimenta, Cláudia Jeane } \\
\text { Lopes; Silva, Cleane Rosa } \\
\text { Ribeiro da; Frazão, Maria } \\
\text { Cristina Lins Oliveira; Almeida, } \\
\text { Francisca das Chagas Alves de. }\end{array}$ & $\begin{array}{l}\text { Contribuição do } \\
\text { enfermeiro ao } \\
\text { aleitamento } \\
\text { materno na atenção } \\
\text { básica }\end{array}$ & $\begin{array}{l}\text { Analisar a contribuição } \\
\text { do enfermeiro para } \\
\text { o aleitamento materno }\end{array}$ & $\begin{array}{l}\text { Contribuições do enfermeiro para a promoção } \\
\text { do aleitamento materno, como instrumento para a } \\
\text { promoção do aleitamento materno. } \\
\text { Promoção de ações voltadas para orientação ainda no } \\
\text { pré-natal identificando quais as dificuldades que } \\
\text { poderão surgir pelas futuras mães para que possam } \\
\text { intervir da melhor maneira possível. }\end{array}$ \\
\hline
\end{tabular}

Fonte: Dados da pesquisa. 


\section{Discursão}

Os dados colhidos nesta revisão mostram claramente que a atuação do enfermeiro frente às informações e orientações às gestantes e puérperas é de grande relevância para um aleitamento materno exclusivo e de sucesso.

Algumas mães ainda desconhecem a grande importância do aleitamento materno exclusivo até os 6 primeiros meses de vida e as patologias mamárias acabam levando ao desmame precoce e é justamente por este motivo que atuação do enfermeiro junto a estas mãe é de grande importância visando uma maior informação e um momento prazeroso e saudável para as mães e os bebês.

Os benefícios de amamentar até os seis meses são muitos, tanto para a criança quanto para a mãe. E estamos descobrindo ganhos ainda mais duradouros que se refletem ao longo de toda a vida. Por meio do leite, a mãe passa ao bebê vários anticorpos que são extremamente importantes para a saúde dele. (Brasil, 2017)

Frente às várias dificuldades enfrentadas pelas mães como a falta de informação adequada, o manejo e pega correta do bico do peito pelo bebê, estão também às dores pós-parto que contribuem muito para a não adesão ao aleitamento materno.

A técnica de amamentação, ou seja, a maneira como a dupla mãe/bebê se posiciona para amamentar/mamar e a pega/sucção do bebê são muito importantes para que o bebê consiga retirar, de maneira eficiente, o leite da mama e também para não machucar os mamilos (Brasil, 2015). Considerando-se também que o profissional de saúde deve atuar junto às mulheres, a fim de que suas ações de promoção à saúde contribuam para o incentivo da amamentação, apoiando-as frente às dificuldades encontradas durante essa prática (Vargas, Alves, Rodrigues, Guerra, Souza \& Branco, 2016).

Por estas razões que a atuação do enfermeiro é imprescindível na vida destas mães, com seu conhecimento técnico teórico e sua visão holística sobre o assunto, ele se torna um profissional chave para o sucesso da amamentação. Dados importantes foram analisados nesta pesquisa sobre a atuação do enfermeiro e estratégias de abordagem junto às mães e demonstraram a eficácia da intervenção e atuação deste profissional, análises de dados confirmaram que a informação e a intervenção adequada surte efeito benéfico nesta situação.

Confirmando os dados acima Souza (2019) relata que o profissional de enfermagem detém um relevante papel na assistência à puérpera, uma vez que desempenha a função de educador, proporcionando a promoção da saúde por meio de suas orientações. (Souza, Alves, Rodrigues, Branco, Oliveira \& Oliveira.). Corroborando Monteschio (2015), considerando a importância do aleitamento materno para a promoção da saúde infantil, reafirma que o enfermeiro é o profissional que mais diretamente pode difundir proteger e apoiar esta prática. (Monteschio, Gavia \& Moreira,2015).

O enfermeiro não é apenas um profissional que acolhe, mas também é aquele que informa, orienta e auxilia as mães e bebês durante o processo de amamentação. Com informações e técnicas precisas e eficazes melhorando a qualidade de vida da mãe e da criança, trabalhando em conjunto para um melhor resultado.

\section{Considerações Finais}

Esta pesquisa demonstrou que o conhecimento acerca do papel do enfermeiro junto ao aleitamento materno exclusivo é de suma importância para mães e bebês, pode-se afirmar que um profissional qualificado é essencial para uma orientação adequada, proporcionando às gestantes e puérperas um conhecimento correto e seguro, e aos bebês um momento prazeroso e com qualidade nutricional. Em um contexto geral pode-se afirmar com veemência que este profissional tem grande valia e importância ao manejo clínico referente ao processo de amamentação.

Demonstrando que conhecimento técnico teórico, juntamente com um olhar profissional que o enfermeiro tem, é de extrema relevância para uma boa orientação para com estas mães. A maternidade é um momento mágico, mas ao mesmo tempo pode ser traumático e assustador para algumas mães, por este motivo ter um profissional qualificado que possa orientar e auxiliar as mães neste momento é essencial. 
E o enfermeiro é sem sombra de dúvidas este profissional, seu conhecimento atrelado ao seu olhar humanizado propicia um atendimento de qualidade e seguro para mães e bebês tornando este momento mais prazeroso e saudável.

\section{Agradecimentos}

Em primeiro quero agradecer a DEUS por sempre estar presente em minhas orações e nunca me faltar nos momentos difíceis e tumultuados, agradeço imensamente a minha família que sempre esteve ao meu lado me apoiando, incentivando para que eu não desistisse e meu agradecimento maior a minha mãe pelas orações incentivos e dedicação a mim, por sempre ter acreditado mais do que eu mesma que um dia eu conseguiria.

\section{Referências}

Alves, T. R. de M., Carvalho, J., Bittencourt, L. de, Lopes, T. R. G., Silva, G. W. dos S., \& Teixeira, G. A. (2018). Contribuições de enfermeiros na promoção do aleitamento materno exclusivo. https://pesquisa.bvsalud.org/portal/resource/pt/biblio-981334

Barreto, C. A., Silva, L. R., \& Cristoffel, M. M. (2009). Aleitamento materno: a visão das puérperas. Rev. Eletr. Enf. 11(3), 605-11

Brasil (2015). Saúde da Criança: Aleitamento Materno e Alimentação Complementar, caderno de atenção básica ${ }^{\circ} 23$ do Ministério da Saúde. Saúde da criança: aleitamento materno e alimentação complementar.

Brasil. (2017). A importância da amamentação até os seis meses. https://saudebrasil.saude.gov.br/eu-quero-me-alimentar-melhor/a-importancia-do-leitematerno-nos-primeiros-seis-meses-da-crianca\#: :text=O\%20 aleitamento $\% 20$ materno\%20 reduz $\% 20 \mathrm{em}$, reduz $\% 20 \mathrm{a} \% 20$ chance $\% 20 \mathrm{de} \% 20$ obesidade.

Carrascoza, K. C., Possobon, R. de F., Costa-Júnior, Á. L., \& Moraes A. B. A. (2011). Aleitamento materno em crianças até os seis meses de vida: percepção das mães. https://www.scielo.br/scielo.php?pid=S0103- 73312011000300015\&script=sci_arttext\&tlng=pt.

Chaves, R.G. Por que amamentar exclusivamente até 6 meses e manter a amamentação até 2 anos ou mais? In: Santiago, L.B. Manual de aleitamento materno. São Paulo: Manole, 2015. https://www.areaseg.com/bib/25\%20-\%20Familia/Manual_Aleitamento_Materno_25NOV_AF.pdf

Costa, E. F. G. da Alves, V. H. Y., Souza, R. de M. P., Rodrigues, D. P., Santos, V. dos \& Oliveira, F. L. (2018). Atuação do enfermeiro no manejo clínico da amamentação: estratégias para o aleitamento materno. https://pesquisa.bvsalud.org/portal/resource/pt/biblio-908417.

Costa, F. dos S. et al (2019). Promoção do aleitamento materno no contexto da estratégia de saúde da família. https://pesquisa.bvsalud.org/portal/resource/pt/biblio-1006281

Ercoli, F. F. et al (2014). Revisão integrativa versus revisão sistemática. https://www.reme.org.br/artigo/detalhes/904

Leal, C. C. G., et al. (2016). Prática de enfermeiras na promoção do aleitamento materno de adolescentes brasileiras. https://pesquisa.bvsalud.org/portal/resource/pt/biblio839759

Lima, C. M., et al (2019). Autoeficácia na amamentação exclusiva: avaliação dos domínios técnica e pensamentos intrapessoais em puérperas. http://revista.cofen.gov.br/index.php/enfermagem/article/view/1597.

Monteschio, C. A. C., et al. (2015). O enfermeiro frente ao desmame precoce na consulta de enfermagem à criança. https://www.scielo.br/scielo.php?script=sci_arttex t\&pid=S0034-71672015000500869.

Moura L. P., Oliveira J. M., \& Noronha, D. D. (2017). Percepção de mães cadastradas em uma estratégia saúde da família sobre aleitamento materno exclusivo. https://periodicos.ufpe.br/revistas/revistaenfermagem/article/viewFile/13983/16836

Núcleo de Telessaúde NUTES PE. (2016). https://aps.bvs.br/aps/quais-os-procedimentos-para-apoiar-oaleitamento-materno-exclusivo/.

Oliveira C. M., santos T. C., \& Melo, I. M. (2017). Promoção do Aleitamento Materno: intervenção educativa no âmbito da Estratégia de Saúde da Família. http://periodicos.pucminas.br/index.php/enfermagemrevista/article/view/16326.

Santos, J. T. (2017). A prevalência do aleitamento materno exclusivo em crianças de 0 a 6 meses. http://tempus.unb.br/index.ph p/tempus/article/view/2197.

Silva, C. L., et al (2014). Intervenção do enfermeiro no aleitamento materno. http://periodicos.fametro.edu.br/index.php/AE/article/view/52.

Silva, L. S. et al (2020). Contribuição do enfermeiro ao aleitamento materno na atenção básica. https://pesquisa.bvsalud.org/portal/resource/pt/biblio-1102780.

Souza, R. de M. P. et al.(2019). O conhecimento do enfermeiro acerca do manejo clínico da amamentação: saberes e práticas. https://pesquisa.bvsalud.org/portal/resource/pt/biblio-968598.

Souza, M. T. et al. (2010). Revisão Integrativa: O que é e como faze.

Vargas. G. S.`A. et al (2016). Atuação dos profissionais de saúde da estratégia saúde da família: Promoção da prática do aleitamento materno. https://periodicos.ufba.br/index.php/enfermagem/article/view/14848 .

Vieira, G. M., et al. (2017). Protocolo de enfermagem para assistência à mulher em processo de lactação. https://pesquisa.bvsalud.org/portal/resource/pt/biblio-908507. 